\title{
Analysis of BAC-end sequences in rainbow trout: Content characterization and assessment of synteny between trout and other fish genomes
}

Carine Genet ${ }^{1 *}$, Patrice Dehais ${ }^{2,3}$, Yniv Palti ${ }^{4}$, Guangtu Gao ${ }^{4}$, Frederick Gavory ${ }^{5}$, Patrick Wincker ${ }^{5}$, Edwige Quillet ${ }^{1}$ and Mekki Boussaha'

\begin{abstract}
Background: Rainbow trout (Oncorhynchus mykiss) are cultivated worldwide for aquaculture production and are widely used as a model species to gain knowledge of many aspects of fish biology. The common ancestor of the salmonids experienced a whole genome duplication event, making extant salmonids such as the rainbow trout an excellent model for studying the evolution of tetraploidization and re-diploidization in vertebrates. However, the lack of a reference genome sequence hampers research progress for both academic and applied purposes. In order to enrich the genomic tools already available in this species and provide further insight on the complexity of its genome, we sequenced a large number of rainbow trout BAC-end sequences (BES) and characterized their contents.

Results: A total of 176,485 high quality BES, were generated, representing approximately $4 \%$ of the trout genome. BES analyses identified 6,848 simple sequence repeats (SSRs), of which 3,854 had high quality flanking sequences for PCR primers design. The first rainbow trout repeat elements database (INRA RT rep1.0) containing 735 putative repeat elements was developed, and identified almost 59.5\% of the BES database in base-pairs as repetitive sequence. Approximately $55 \%$ of the BES reads $(97,846)$ had more than 100 base pairs of contiguous non-repetitive sequences. The fractions of the 97,846 non-repetitive trout BES reads that had significant BLASTN hits against the zebrafish, medaka and stickleback genome databases were 15\%,16.2\% and 17.9\%, respectively, while the fractions of the non-repetitive BES reads that had significant BLASTX hits against the zebrafish, medaka, and stickleback protein databases were 10.7\%, 9.5\% and 9.5\%, respectively. Comparative genomics using paired BAC-ends revealed several regions of conserved synteny across all the fish species analyzed in this study.

Conclusions: The characterization of BES provided insights on the rainbow trout genome. The discovery of specific repeat elements will facilitate analyses of sequence content (e.g. for SNPs discovery and for transcriptome characterization) and future genome sequence assemblies. The numerous microsatellites will facilitate integration of the linkage and physical maps and serve as valuable resource for fine mapping QTL and positional cloning of genes affecting aquaculture production traits. Furthermore, comparative genomics through BES can be used for identifying positional candidate genes from QTL mapping studies, aid in future assembly of a reference genome sequence and elucidating sequence content and complexity in the rainbow trout genome.
\end{abstract}

\footnotetext{
* Correspondence: carine.genet@jouy.inra.fr

'INRA, UMR 1313 GABI, Génétique Animale et Biologie Intégrative, 78350

Jouy-en-Josas, France

Full list of author information is available at the end of the article
} 


\section{Background}

Rainbow trout (Oncorhynchus mykiss) are cultivated worldwide for aquaculture production. Trout farming has been successful in North America, the species native area, as well as in many other regions, including Chile and a number of European countries where rainbow trout had been introduced since the $19^{\text {th }}$ century. In 2008 , total world production was about 576,000 metric tons with a total export value estimated around 2.4 billions USD (http://www.fao.org/fishery/statistics/en).

The rainbow trout is one of the most intensively studied fish species. Several features such as in vitro fertilization, ease of rearing and gamete handling and a large body size with large and clearly defined tissues, make it a particularly suited model to carry out a range of investigations. Hence, considerable amount of basic knowledge has been accumulated in many areas such as physiology, nutrition, behaviour, ecology, genetics, pathology, comparative immunology, carcinogenesis and toxicology (reviewed in [1]).

Combining biological and phenotypic data with genomic information can be used to increase our basic knowledge of the regulation of biological functions, and ultimately used in applied research to improve the environmental and genetic management of aquaculture production systems with focus on complex traits such as meat and carcass quality, stress tolerance or resistance to specific pathogens.

The rainbow trout genome size was estimated to be between 2.4 and $3.0 \times 10^{9}$ base pairs (bp) [2]. A whole genome duplication event occurred 25 to 100 million years ago in the common ancestor of the salmonids. Since that time, re-diploidization has resulted in a semitetraploid state [3]. Consequently, presence of duplicated genetic markers was reported [4] and many homeologous regions have been identified in the rainbow trout genome [5]. Although the tetraploidization event increased the genome complexity, it also makes the salmonids a very pertinent group to study the differential evolution and loss of duplicated genes in the process of re-diploidization.

Several genomic resources have been developed in rainbow trout in the last decade. Seven linkage maps based on either AFLP markers [2,6] or microsatellite markers and few SNPs [7-11] have been constructed. These maps are used for comparative mapping across salmonid species [12], for QTL mapping studies for various traits [13-20] or for linkage disequilibrium studies [21]. Attempts for high throughput discovery of SNP markers are emerging but only a limited number of true SNP have been validated up to now [22]. Large EST databases ([23,24]; http://compbio.dfci.harvard.edu/cgibin/tgi/gimain.pl?gudb=r_trout and http://www.sigenae. org) are available, as well as high content DNA microarrays $[25,26]$. Several bacterial artificial chromosome (BAC) libraries have also been established $[4,27,28]$.

BAC libraries are a valuable genomic resource for many purposes, including clone-based sequencing, positional cloning and physical mapping. The first physical map in rainbow trout was recently built using the 10X HindIII BAC library [28]. The map contained 4,173 contigs and 9,379 singletons. The physical length of the map contigs was estimated to be approximately $2.0 \mathrm{~Gb}$, which represents almost $83 \%$ of rainbow trout genome.

BAC-end sequencing has been initially proposed to be an efficient approach for whole genome sequencing projects [29], for comparative physical mapping [30,31], and for the development of molecular markers, mainly microsatellites [32]. In the absence of whole genome sequences, BES analysis can elucidate sequence content and complexity, including gene density, potential transposable elements, and microsatellite markers [33]. Furthermore, paired BAC-end sequences can be very useful for scaffolding in whole-genome sequencing assembly projects.

Here we report on the sequencing and characterization of BAC-end sequences (BES) from more than half of the clones from the rainbow trout 10X HindIII BAC library. The sequence content was analysed for putative genes, repetitive elements and simple sequence repeats (SSR). BES gene content was then used to establish regions of conserved synteny with other fish genomes.

\section{Results and Discussion \\ BAC-end sequencing statistics}

Sequencing of rainbow trout BAC ends generated 177,857 raw data reads of more than 100 bp from 92,593 BAC clones, of which 85,120 ( 92\%) had both ends sequenced and 7,473 had only one end sequenced. An additional 1,372 BES were filtered-out because of high similarity to bacterial and vector sequences or because of low quality sequence. The total of high quality rainbow trout sequence reads was 176,485 including one hundred forty eight BES sequenced twice for quality control purposes.

The PHRED Q20 read length ranged from 101 to 832 bp with a mean of $546 \mathrm{bp}$. The trout BES Q20 average length was similar to catfish [34] but lower than Atlantic Salmon [35]. Overall, BAC-end sequencing generated a total $96,298,179$ bp of genomic sequence representing approximately $4 \%$ of the trout genome (assuming genome size of $2.4 \times 10^{9} \mathrm{bp}$ ). The GC content was estimated to be around $42 \%$, which is lower than channel catfish [34] and stickleback (44\%), but higher than zebrafish (36\%) and medaka (40\%) (http://genome.ucsc.edu). 


\section{Preliminary survey of repeat content from rainbow trout genome}

Few studies have reported the identification and characterization of repeat elements in salmonids, resulting in the absence of sufficient repeat masking data set for rainbow trout. Repbase update release 13.05 contains only 145 ancestral shared repeats and one lineage-specific repeat for rainbow trout, and for salmon, 141 ancestral shared repeats and five lineage-specific repeats [36]. Consequently, masking of rainbow trout BES using RepBase 13.05 generated only $1.66 \%$ of masked sequences. Therefore, we used the BES data to generate a new rainbow trout repeat library. This database contains 735 putative elements and was named INRA RT rep1.0 (available as Additional file 1). Repeat element analysis using the new INRA RT rep1.0 library masked almost $59.5 \%$ of the BES database in base-pairs (data not shown). The most abundant repeat elements were DNA transposons, and the most common transposon type was the TcMar-Tc1 transposon-related sequence (24.5\%) (Table 1). Unknown elements were also abundant and accounted for $19.2 \%$ of BES (Table 1).

For comparison we masked the rainbow trout BES dataset with the Atlantic salmon repeat database (http://web. uvic.ca/grasp and [37]) and found that only $52.3 \%$ of the BES dataset was masked compared to $59.5 \%$ with the rainbow trout repeat database. We also estimated the redundancy of each species specific database by repeatmasking the INRA rep1.0 by Salmon Raw 1.6 database and vice versa. We observed that respectively $35.3 \%$ and $52.8 \%$ of the databases were masked suggesting differences in the repeats content between the two salmonid species. However, it is also possible that the restriction enzyme selection for the BAC libraries preparation (EcoRI for Atlantic salmon and HindIII for rainbow trout) might have imposed some bias on the repeats content of each database.

In addition to the automated detection of repeats using RepeatModeler, we checked for undetected repetitive elements by aligning the masked BES reads to each other. The alignment results were classified according to the number of significant hits and are summarized in Figure 1. Most of the BES reads (93.4\%) had less than 10 hits, indicating that masking with the INRA RT rep1.0 library was effective. However, 711 reads $(6.6 \%)$ produced more than 10 hits, suggesting that these BES reads may contain repetitive DNA sequences. Moreover, almost 263 reads (2.4\%) produced more than 50 hits, suggesting that these BES reads contain interspersed repeats not yet in INRA RT rep1.0 database.

Some classes of repeat elements in salmonids can be as long as 1,500 bp [37]. Progress is currently being made to further characterise these long interspersed repeats in rainbow trout by using full length BAC-insert sequencing which will enable characterizing full-length copies of repeat elements and identifying new repeats (Jean-Nicolas Volff, personal communication).

Table 1 Characteristics of the INRA RT rep1.0 database

\begin{tabular}{|c|c|c|c|c|c|}
\hline & $\begin{array}{l}\text { Number of } \\
\text { elements }\end{array}$ & $\begin{array}{l}\text { Percentage of } \\
\text { sequence }\end{array}$ & & $\begin{array}{l}\text { Number of } \\
\text { elements }\end{array}$ & $\begin{array}{l}\text { Percentage of } \\
\text { sequence }\end{array}$ \\
\hline \multicolumn{3}{|l|}{ Class I elements } & \multicolumn{3}{|l|}{ Class II elements } \\
\hline LTR retrotransposons & & & DNA & 1 & 0,05 \\
\hline LTR/DIRS1 & 2 & 0,02 & DNA/Harbinger & 2 & 0,02 \\
\hline LTR/Gypsy & 21 & 1,68 & DNA/hAT & 9 & 0,27 \\
\hline $\begin{array}{l}\text { Non-LTR } \\
\text { retrotransposons }\end{array}$ & & & DNA/hAT-Charlie & 5 & 0,26 \\
\hline LINE & 1 & 0,29 & DNA/hAT-Tag1 & 1 & 0,05 \\
\hline LINE/I & 1 & 0,01 & DNA/hAT-Tip100 & 1 & 0,01 \\
\hline LINE/Jockey & 3 & 0,05 & DNA/Helitron & 1 & 0,01 \\
\hline LINE/L1 & 6 & 0,14 & DNA/Maverick & 1 & 0,01 \\
\hline LINE/L2 & 19 & 2,18 & DNA/TC1 & 11 & 4,26 \\
\hline LINE/Penelope & 1 & 0,05 & DNA/TcMar-Tc1 & 46 & 24,51 \\
\hline LINE/Rex1 & 6 & 3,03 & DNA/TcMar-Tc2 & 1 & 0,01 \\
\hline SINE & 5 & 1,09 & Others elements & & \\
\hline SINE/Deu & 5 & 0,23 & Simple_repeat & 22 & 1,19 \\
\hline SINE/tRNA-Lys & 1 & 0,01 & rRNA & 3 & 0,11 \\
\hline SINE/5S & 1 & 0,01 & Satellite & 2 & 0,06 \\
\hline SINE? & 8 & 0,37 & $\begin{array}{l}\text { Unknown } \\
\text { elements }\end{array}$ & 549 & 19,19 \\
\hline
\end{tabular}

Columns 1 and 4 show the name of the repeat elements. Columns 2 and 5 provide the number of repeats elements in the INRA RT rep1.0 generated in this work. Columns 3 and 6 correspond to the percentage of total base pairs masked by the corresponding repeat element. 


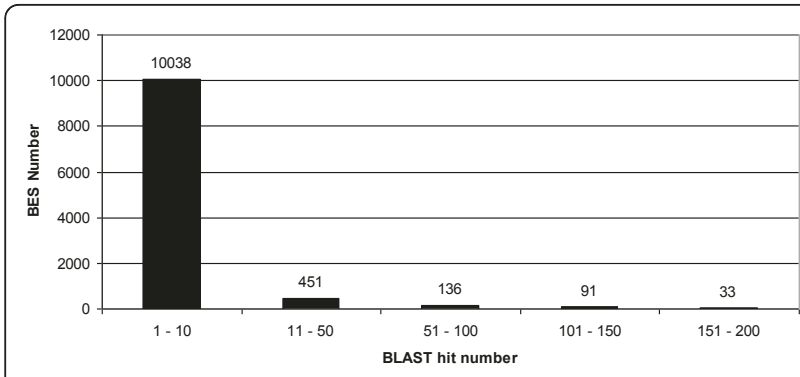

Figure 1 Low complexity sequences identified through BLAST search of masked BES against themselves. $\times$ axis represents the distribution of BLAST hits. Y axis represents the number of BES.

\section{Development of microsatellite markers}

A total of 6,848 microsatellites were identified in 6,196 BES reads (Table 2). Approximately $56 \%$ of the microsatellites $(3,854)$ were suitable for PCR primers design as they were flanked by sequences of at least $50 \mathrm{bp}$. We were able to define 2,061 primer-pairs $(\sim 30 \%)$ from 1,923 distinct BES as more than one microsatellite can be detected in a single BES.

Di-nucleotides were the most abundant repeat motif (65.1\%) followed by tetra-nucleotides (10.3\%) (Table 2). The most abundant di-nucleotide repeats were TG/CA accounting for $19.5 \%$. AC/GT and $\mathrm{GA} / \mathrm{TC}$ repeats

Table 2 Distribution of Simple Sequence Repeats in trout BAC end sequences.

\begin{tabular}{|c|c|c|c|c|c|}
\hline Repeat & Type & Number & Repeat & Type & Number \\
\hline \multirow[t]{2}{*}{ Monomer } & $\mathrm{A} / \mathrm{T}$ & 71 & Tetramer & CACA/TGTG & 38 \\
\hline & $C / G$ & 1 & & TCTG/CAGA & 37 \\
\hline \multirow[t]{6}{*}{ Dimer } & $\mathrm{TG} / \mathrm{CA}$ & 1346 & & TATG/CATA & 26 \\
\hline & $\mathrm{AC} / \mathrm{GT}$ & 1238 & & AGAC/GTCT & 38 \\
\hline & $\mathrm{GA} / \mathrm{TC}$ & 607 & & GAGA/TCTC & 24 \\
\hline & $\mathrm{TA} / \mathrm{TA}$ & 505 & & ACAT/ATGT & 22 \\
\hline & $\mathrm{AG} / \mathrm{CT}$ & 498 & & AGAA/TTCT & 17 \\
\hline & AT/AT & 311 & & Other & 370 \\
\hline \multirow[t]{6}{*}{ Trimer } & $\mathrm{AAC/GTT}$ & 5 & Pentamer & $\begin{array}{l}\text { TCAAA } \\
\text { TITGA }\end{array}$ & 19 \\
\hline & CAT/ATG & 13 & & $\begin{array}{l}\text { ATTTG/ } \\
\text { CAAAT }\end{array}$ & 15 \\
\hline & AAT/ATT & 36 & & Other & 92 \\
\hline & ATA/TAT & 36 & Hexamer & & 356 \\
\hline & TTA/TAA & 24 & Heptamer & & 80 \\
\hline & Other & 55 & Octomer & & 278 \\
\hline \multirow[t]{4}{*}{ Tetramer } & $\begin{array}{l}\text { ACAC/ } \\
\text { GTGT }\end{array}$ & 26 & Nanomer & & 144 \\
\hline & $\begin{array}{l}\text { TGTA/ } \\
\text { TACA }\end{array}$ & 66 & Decamer & & 478 \\
\hline & $\begin{array}{l}\text { ATAC/ } \\
\text { GTAT }\end{array}$ & 23 & & & \\
\hline & $\begin{array}{l}\text { CTCA/ } \\
\text { TGAG }\end{array}$ & 25 & Total & & 6,920 \\
\hline
\end{tabular}

accounted for $17.9 \%$ and $8.8 \%$, respectively. Polymorphism and usefulness of the BES microsatellites for linkage analysis and genetic mapping was assessed in the USDA-NCCCWA panel of five families [9] using 193 markers (Additional file 2). Out of the 193 loci tested, $126(65.3 \%)$ were polymorphic with allele numbers ranging from 2 to 12; 57 (29.5\%) failed to produce a specific PCR product and 10 (5.2\%) were monomorphics. Fifteen microsatellite loci were duplicated, of which nine were useful for linkage mapping. These results show that most of the microsatellite markers developed from BES are polymorphic in the NCCCWA reference families and thus can be used for direct integration between the genetic and physical maps of the rainbow trout genome [38].

\section{Homology with other fish genomes}

We investigated the BES sequence homology and gene content by computational and manual annotations. Of the 176,485 high quality BES reads, 97,846 BES reads (55\%) had more than 100 base pairs of contiguous non repetitive sequences and were used for assessing genome syntenies by BLASTN and BLASTX similarity searches against the ENSEMBL genome and peptide databases of zebrafish, medaka and stickleback. The fractions of the 97,846 non-repetitive trout BES reads that had significant BLASTN hits against the zebrafish, medaka and stickleback genome databases were $15 \%, 16.2 \%$ and $17.9 \%$, respectively, while the fractions of the non-repetitive BES reads that had significant BLASTX hits against the zebrafish, medaka, and stickleback protein databases were $10.7 \%, 9.5 \%$ and $9.5 \%$, respectively (Tables 3 and 4). Compared to similar analyses that used BES from non-model fish species to construct "In-silico" comparative genome maps [39-41], the sequence homologies between rainbow trout and the model species were low,

Table 3 Rainbow trout BAC end sequences BlastN statistics

\begin{tabular}{|c|c|c|c|}
\hline & Medaka & Stickleback & Zebrafish \\
\hline Total BES with hits & 15,896 & 17,499 & 14,716 \\
\hline BES with unique hits ${ }^{a}$ & 15,379 & 15,612 & 13,868 \\
\hline \multicolumn{4}{|l|}{ BACs } \\
\hline BAC with one end & 14,206 & 15,467 & 13,304 \\
\hline Paired BAC-ends & 845 & 1,016 & 706 \\
\hline $\begin{array}{l}\text { Paired BAC-ends with unique } \\
\text { hits }\end{array}$ & 792 & 812 & 632 \\
\hline $\begin{array}{l}\text { Unique hits paired BAC-ends } \\
\text { matched on the same } \\
\text { chromosome }\end{array}$ & 418 & 566 & 360 \\
\hline Also identified by BlastX & 85 & 105 & 105 \\
\hline Microsynteny identified & 307 & 421 & 176 \\
\hline
\end{tabular}

a The bioinformatic filtering criteria used for identifying 'unique' hits are described in the methods section. 
Table 4 Rainbow trout BAC end sequences BlastX statistics

\begin{tabular}{|c|c|c|c|}
\hline & Medaka & Stickleback & Zebrafish \\
\hline Total BES with hits & 9,312 & 9,308 & 10,487 \\
\hline BES with unique hits ${ }^{a}$ & 9,125 & 9,058 & 9,983 \\
\hline \multicolumn{4}{|l|}{ BACs } \\
\hline BAC with one end & 8,700 & 8,674 & 9,717 \\
\hline Paired BAC-ends & 306 & 317 & 385 \\
\hline $\begin{array}{l}\text { Paired BAC-ends with unique } \\
\text { hits }\end{array}$ & 293 & 305 & 355 \\
\hline $\begin{array}{l}\text { Unique hits paired BAC-ends } \\
\text { matched on the same } \\
\text { chromosome }\end{array}$ & 132 & 148 & 155 \\
\hline Also identified by BlastN & 85 & 105 & 105 \\
\hline Microsynteny identified & 40 & 45 & 30 \\
\hline
\end{tabular}

a The bioinformatic filtering criteria used for identifying 'unique' hits are described in the methods section.

most likely due to the large evolutionary distance between the salmonids and the model species.

Multiple gene hits may be caused by gene duplications or by the presence of conserved sequences among members of gene families. BLASTX searches revealed hits with some genes existing in large copy numbers as exemplified by protocadherin members families (53 hits) and odorant receptor members families (48 hits) (Additional file 3). The others gene hits showed identity to transposable elements such as piggybac transposable element 4 or LINE-1 type transposase domain containing 1 or transposase (data not shown) which revealed that these transposable elements were not masked by the INRA RT rep1.0 database.

\section{Identification of regions of microsynteny}

The sequence homology searches were conducted using both BlastN and BlastX alignment tools.

For BlastN searches, we identified 792, 812, and 632 BACs with both ends having significant unique hits to the medaka, stickleback, and zebrafish genomes, respectively (Table 3). Out of those, 418 (53\%), 566 (70\%), and $360(57 \%)$ unique paired BAC-end hits matched on the same chromosome (macro-synteny) in medaka, stickleback and zebrafish, respectively. Of those, we identified 307 (73\%), 421 (74\%) and 176 (49\%) regions of microsynteny between rainbow trout and medaka, stickleback, and zebrafish, respectively (additional file 4).

For BLASTX analysis, we identified 293, 305, and 355 BACs with both ends having significant gene hits to the medaka, stickleback, and zebrafish genomes, respectively (Table 4). Further analysis revealed that 132 (45\%; medaka), 148 (48\%; stickleback), and 155 (43\%; zebrafish) paired BAC-end gene hits were in macro-synteny. Of those, we identified $40(30 \%), 45(30 \%)$ and $30(30 \%)$ regions of microsynteny between rainbow trout and medaka, stickleback, and zebrafish, respectively (additional file 5). Finally, our BlastN analyses identified 85 (medaka), 105 (stickleback), and 105 (zebrafish) unique paired BAC end hits in macrosynteny that were also detected using BlastX analyses (Table 3).

As expected, our analysis revealed moderate macrosynteny between rainbow trout and the three model species and even lower level of microsynteny likely due to chromosomal rearrangements that have occurred since the divergent of those species from a common teleost ancestor. The strongest decline in number of microsynteny regions was observed for zebrafish, which is indeed more distant from rainbow trout than the medaka and stickleback [42]. While the number of significant BES hits with BlastN was between 1.78 (zebrafish) to 2.7 (stickleback) times greater than those with BlastX hits, the difference in identifying microsynteny was even greater. The number of microsynteny regions identified with BlastN was 5.8 (zebrafish) to 9.3 (stickleback) times greater than BlastX. This can be explained by several factors including incomplete annotations of the model fish genomes and the presence of pseudogenes and conserved non-coding sequences that were not included in the peptide databases. BlastN also appears to be more accurate for estimating microsynteny as it provides the exact points of sequence matches on the chromosomes of the reference genomes (instead of the ORF boundaries for BlastX). However, it is also important to note that $17 \%$ (stickleback) to 33\% (zebrafish) of the macrosynteny BAC paired ends identified by BlastX were not revealed by BlastN. This may be caused by non- or lessconserved peptides whose coding sequences are not under strong selection pressure and have evolved enough to escape detection as significant unique hits by BlastN.

The comparative genome analyses reported here provide a survey of conserved synteny between rainbow trout and three model fish species. The results of our analyses suggest that for many regions in the rainbow trout genome comparative mapping might serve as a useful genomic resource for identifying candidate genes in QTL detection studies. Nevertheless, further assessment of regions of conserved synteny by direct sequencing of full-length BAC clones and by evaluating gene content and orthology revealed that gene order, orientations, and gene length are highly variable across fish species (manuscript in preparation).

\section{Conclusions}

In the present study, we sequenced and subsequently characterized more than half of BAC ends from the rainbow trout Swanson YY double haploid male 10X HindIII BAC library. These genomic sequences were used to generate the first rainbow trout specific repeat 
database containing 735 putative repeat elements. This database is useful for repeat masking in salmonid genomes. Approximately 59.5\% of the BES database in base pairs was masked by this repeat database, providing for the first time an estimate of how much of the rainbow trout genome is composed of repetitive sequences. We detected 6,848 microsatellites in the BES dataset, of which 3,854 presented high quality flanking sequences with more than $50 \mathrm{bp}$ in length. A subset of those were validated and used to construct the first rainbow trout integrated genetic-physical map [38]. The development of those new microsatellite markers will also serve to increase marker densities on current rainbow trout genetic maps and initiate in silico comparative mapping with species whose genomes have been fully sequenced. Paired BAC-ends were used to establish regions of microsynteny between trout and model fish species (zebrafish, medaka, and stickleback). The microsynteny analyses revealed low to intermediate genome homology between rainbow trout and the other fish species. Our findings suggest that due to chromosomal breakage and rearrangements that have occurred during fish genomes evolution, only closely related species like other salmonids will be useful for chromosome-wide and genomewide comparative analyses with rainbow trout.

\section{Methods}

\section{BAC culture and BAC-end sequencing}

A 10X HindIII bacterial artificial chromosome (BAC) library from a Swanson YY male doubled haploid homozygous line was previously constructed [4]. More than half of this library $(99,840$ BAC clones) was used for BAC-end sequencing. BAC culture and sequencing reactions were conducted, as described previously [43]. Briefly, BAC DNA was extracted using a standard alkaline lysis protocol developed by Genoscope (Evry, France). Sequencing reactions were carried out in the Genoscope facility with T7 or Sp6 universal primers, using ABI kit version 3.1. Generated raw sequence files were subsequently processed using the PHRED software [44], vector and bacterial sequences were removed. Q20 values were achieved by setting the sequence quality PHRED score cut-off value to 20 . All processed BES were submitted to the EMBL/EBI database with consecutive accession numbers of FQ482162-FQ658498 and are available through the web site of the INRA bioinformatics group (http://www.sigenae.org/troutBES).

\section{Identification of repetitive DNA elements Complex DNA repetitive elements}

RepeatModeler software was used for identifying repeat elements boundaries and for classifying the newly reconstructed repetitive sequence from the rainbow trout BES data (http://www.repeatmasker.org/RepeatModeler.html).
A specific rainbow trout repeat database was constructed and was named INRA RT Rep1.0. This database was subsequently used as a custom file for masking BES sequences using RepeatMasker (http://www.repeatmasker.org/).

The Atlantic salmon repeat database was used (file Salmon Raw Repeat DB V1_6 available at http://web. uvic.ca/grasp) for comparison purposes.

Identification of microsatellites and simple sequence repeats Microsatellites and other SSR motifs were identified using Tandem Repeat Finder (TRF) software [45]. We examined ten classes of SSRs by using a maximum period size of 10 with default settings. BES containing microsatellites were subsequently masked using RepeatMasker with INRA RT rep1.0 custom library file: BES harbouring SSRs with at least 50 bp flanking sequences were then selected and forward and reverse primers were designed using Primer3 software [46]. Microsatellites and corresponding flanking sequences were submitted to the GenBank STS database with consecutive accession numbers of GF100674-GF100698; GF107484GF107651; GF107921-GF109647; GF110457-GF110594 and GF110820-GF110822.

\section{Assessment of microsatellites polymorphism}

The polymorphism of 193 microsatellites markers was assessed by genotyping 10 parents from the National Center for Cool and Cold Water Aquaculture (NCCCWA) reference mapping panel [9]. Primers were optimized for amplification by varying annealing temperatures and $\mathrm{MgCl}_{2}$ concentrations. PCR amplifications were conducted in an MJ Research DNA Engine thermal cycler model PTC 200 (MJ Research, Waltham, MA) as previously described [47]. Three microliters of each PCR product were added to $20 \mu \mathrm{L}$ of water, $1 \mu \mathrm{L}$ of the diluted sample was added to $12.5 \mu \mathrm{L}$ of loading mixture made up with $12 \mu \mathrm{L}$ of $\mathrm{HiDi}$ formamide and $0.5 \mu \mathrm{L}$ of Genscan 400 ROX internal size standard. Samples were denatured at $95{ }^{\circ} \mathrm{C}$ for $5 \mathrm{~min}$ and kept on ice until loading on an ABI 3730 DNA Analyzer (ABI, Foster City, $\mathrm{CA})$. Output files were analyzed using GeneMapper version 3.7 (ABI, Foster City, CA),

\section{Assessment of regions of synteny with other fish genomes \\ Sequence homology searches and results filtration}

Masked BES reads with more than 100 base pairs of contiguous non-repetitive sequences were analysed for sequence homology by BLASTN using ENSEMBL DNA databases for zebrafish (Danio_rerio.Zv9.61.dna_rm.toplevel.fa), stickleback (Gasterosteus_aculeatus.BROADS1.61. dna_rm.toplevel.fa), and medaka (Oryzias_latipes. MEDAKA1.61.dna_rm.toplevel.fa) and for gene content by BLASTX using the ENSEMBL non redundant protein 
databases for zebrafish (Danio_rerio.Zv9.61.pep.all.fa), stickleback (Gasterosteus_aculeatus.BROADS1.61.pep.all. fa), and medaka (Oryzias_latipes.MEDAKA1.61.pep.all.fa). BLASTN and BLASTX searches were carried out using an e-value cut off of $1 \mathrm{e}^{-5}$ with following parameters (-m9 -r1 -q-1 -G4 -E2 -W9 -F “m D” -U for BLASTN). The BLAST search results were filtered to remove non specific sequences using the following filtration steps: (1) For each BES read with BLAST hit, results were filtered to keep only the hits with the minimal e-value score; (2) BES reads with at least two hits having the same minimal e-value were filtered to keep the hits with the highest HSPs (highscoring segment pairs; calculated as the product of \% identity multiplied by alignment length); and 3) keep only BES reads with single hits following filtration steps 1 and 2. For BLASTX the Ensembl protein IDs were renamed by their corresponding Ensembl gene IDs as each gene may encode several peptides due to alternative splicing.

\section{Comparative synteny analysis}

Identification of regions of conserved synteny between rainbow trout and model fish species were investigated using paired BAC-ends with unique hits. A region of microsynteny with the target genome was established if both BAC ends were mapped to the same chromosome with a space of 10 to $300 \mathrm{~Kb}$ between both ends and if they were properly oriented (tail-to-tail; the two ends in opposing orientation with 3 ' ends internal) $[41,48,49]$. In addition, we defined regions of macro-synteny as those in which the two paired BAC-end hits were mapped to the same chromosome of the model species.

\section{Additional material}

Additional file 1: INRA RT rep1.0. The first Rainbow trout repeat database elements contains 735 putative elements in fasta format.

Additional file 2: PCR conditions, allele size range, number of alleles and GenBank accession numbers for tested microsatellites. Duplicated markers are in bold. ND indicates that for these duplicated microsatellite it was not possible to determine the allele numbers. * indicates monomorphic or non informative microsatellites in the mapping panel. Abbreviations: Atp: Annealing temperature.

Additional file 3: An example of multiple gene hits corresponding to odorant receptor gene family (sheet 1 ) or protocadherin gene family (sheet 2) in zebrafish.

Additional file 4: Summary of conserved microsyntenies identified by Blastn analysis with medaka (sheet 1 ), stickleback (sheet 2 ) and zebrafish (sheet 3 ). BAC, Forward and reverse hits indicate the orientation of the BES using the forward T7 and the reverse Sp6 sequencing primers, respectively. Span indicates the average distance between the two genes in the corresponding species.

Additional file 5: Summary of conserved microsyntenies identified by BlastX analysis with medaka (sheet 1 ), stickleback (sheet 2) and zebrafish (sheet 3 ) gene hits. Putative identities of mate paired genes were provided as ENSEMBL protein ID. Forward and reverse hits indicate the orientation of the BES using the forward T7 and the reverse Sp6 sequencing primers, respectively. Span indicates the average distance between the two genes in the corresponding species.

\section{Acknowledgments and Funding}

The authors thank CRB GADIE (Diane Esquerre) for BAC clones handling and Brian Smith, Renee Fincham and Kristy Shewbridge for microsatellites genotyping. We are grateful to Yann Guiquen for help and precious contribution to fund raising for this study. This work was made possible by financial support of Genoscope. We acknowledge René Guyomard for his initial contribution in BAC library procurement. The microsatellites genotyping was supported by NRI Grant No. 2007-35616-17875 from the USDA National Institute of Food and Agriculture.

\section{Author details}

'INRA, UMR 1313 GABI, Génétique Animale et Biologie Intégrative, 78350 Jouy-en-Josas, France. ${ }^{2}$ INRA, UMR 444 ENVT Génétique Cellulaire, 31326 Castanet-Tolosan, France. ${ }^{3}$ INRA, Sigenae, 31326 Castanet-Tolosan, France. ${ }^{4}$ National Center for Cool and Cold Water Aquaculture, ARS-USDA, 11861 Leetown Road, Kearneysville, WV 25430, USA. ${ }^{5}$ CEA Genoscope, 2 rue Gaston Crémieux, 91057 Evry Cedex, France.

\section{Authors' contributions}

CG conceived the project. MB and CG supervised the project, and wrote the manuscript. MB and PD carried out bioinformatics analysis. YP performed the microsatellites genotyping analyses, provided 7,296 BAC clones for BAC end Sequencing and revised the manuscript draft. GG participated in and contributed ideas to the bioinformatics analysis. FG and PW carried out DNA extraction and DNA sequencing. EQ helped with interpretation of data analysis and revised the manuscript draft. All authors read, commented on and approved the final manuscript

Received: 3 December 2010 Accepted: 14 June 2011 Published: 14 June 2011

\section{References}

1. Thorgaard GH, Bailey GS, Williams D, Buhler DR, Kaattari SL, Ristow SS, Hansen JD, Winton JR, Bartholomew JL, Nagler JJ, et al: Status and opportunities for genomics research with rainbow trout. Comp Biochem Physiol B Biochem Mol Biol 2002, 133:609-646.

2. Young WP, Wheeler PA, Coryell VH, Keim P, Thorgaard GH: A detailed linkage map of rainbow trout produced using doubled haploids. Genetics 1998, 148:839-850

3. Allendorf FW, Thorgaard GH, Turner BJ: Tetraploidy and the evolution of salmonid fishes. Evolutionary Genetics of Fishes New York: Plenum; 1984 $1-53$.

4. Palti Y, Gahr SA, Hansen JD, Rexroad CE: Characterization of a new BAC library for rainbow trout: evidence for multi-locus duplication. Anim Genet 2004, 35:130-133.

5. Danzmann RG, Davidson EA, Ferguson MM, Gharbi K, Hoyheim B, Koop BF, Lien S, Lubieniecki KP, Moghadam HK, Park J, et al: Distribution of ancestral proto-Actinopterygian chromosome arms within the genomes of $4 \mathrm{R}$-derivative salmonid fishes (Rainbow trout and Atlantic salmon). BMC Genomics 2008, 9:557.

6. Nichols KM, Young WP, Danzmann RG, Robison BD, Rexroad C, Noakes M, Phillips RB, Bentzen P, Spies I, Knudsen K, et al: A consolidated linkage map for rainbow trout (Oncorhynchus mykiss). Anim Genet 2003, 34:102-115.

7. Danzmann RG, Cairney M, Davidson WS, Ferguson MM, Gharbi K, Guyomard R, Holm LE, Leder E, Okamoto N, Ozaki A, et al: A comparative analysis of the rainbow trout genome with 2 other species of fish (Arctic charr and Atlantic salmon) within the tetraploid derivative Salmonidae family (subfamily: Salmoninae). Genome 2005, 48:1037-1051.

8. Guyomard R, Mauger S, Tabet-Canale K, Martineau S, Genet C, Krieg F, Quillet E: A type I and type II microsatellite linkage map of rainbow trout (Oncorhynchus mykiss) with presumptive coverage of all chromosome arms. BMCGenomics 2006, 7:302.

9. Rexroad CE, Palti Y, Gahr SA, Vallejo RL: A second generation genetic map for rainbow trout (Oncorhynchus mykiss). BMC Genet 2008, 9:74.

10. Rexroad CE, Rodriguez MF, Coulibaly I, Gharbi K, Danzmann RG, Dekoning J, Phillips R, Palti Y: Comparative mapping of expressed sequence tags containing microsatellites in rainbow trout (Oncorhynchus mykiss). BMC Genomics 2005, 6:54. 
11. Sakamoto T, Danzmann RG, Gharbi K, Howard P, Ozaki A, Khoo SK, Woram RA, Okamoto N, Ferguson MM, Holm LE, et al: A microsatellite linkage map of rainbow trout (Oncorhynchus mykiss) characterized by large sex-specific differences in recombination rates. Genetics 2000, 155:1331-1345.

12. Gharbi K, Gautier A, Danzmann RG, Gharbi S, Sakamoto T, Hoyheim B, Taggart JB, Cairney M, Powell R, Krieg F, et al: A linkage map for brown trout (Salmo trutta): chromosome homeologies and comparative genome organization with other salmonid fish. Genetics 2006, 172:2405-2419.

13. Barroso RM, Wheeler PA, LaPatra SE, Drew RE, Thorgaard GH: QTL for IHNV resistance and growth identified in a rainbow (Oncorhynchus mykiss) $\times$ Yellowstone cutthroat (Oncorhynchus clarki bouvieri) trout cross. Aquaculture 2008, 277:156-163.

14. Drew RE, Schwabl H, Wheeler PA, Thorgaard GH: Detection of QTL influencing cortisol levels in rainbow trout (Oncorhynchus mykiss). Aquaculture 2007, 272:S183-S194.

15. Haidle $L$, Janssen JE, Gharbi K, Moghadam HK, Ferguson MM, Danzmann RG: Determination of quantitative trait loci (QTL) for early maturation in rainbow trout (Oncorhynchus mykiss). Mar Biotechnol (NY) 2008, 10:579-592.

16. Martinez V, Thorgaard G, Robison B, Sillanpaa MJ: An application of Bayesian QTL mapping to early development in double haploid lines of rainbow trout including environmental effects. GenetRes 2005, 86:209-221.

17. O'Malley KG, Sakamoto T, Danzmann RG, Ferguson MM: Quantitative trait loci for spawning date and body weight in rainbow trout: testing for conserved effects across ancestrally duplicated chromosomes. JHered 2003, 94:273-284.

18. Ozaki A, Khoo SK, Yoshira Y, Ototake M, Sakamoto T, Dijkstra JM, Okamoto N: Identification of additional quantitative trait loci (QTL) responsible for susceptibility to infectious pancreatic virus in rainbow trout. Fish Pathology 2007, 42:131-140

19. Rodriguez MF, LaPatra S, Williams S, Famula T, May B: Genetic markers associated with resistance to infectious hematopoietic necrosis in rainbow and steelhead trout (Oncorhynchus mykiss) backcrosses. Aquaculture 2004, 241:93-115.

20. Zimmerman AM, Evenhuis JP, Thorgaard GH, Ristow SS: A single major chromosomal region controls natural killer cell-like activity in rainbow trout. Immunogenetics 2004, 55:825-835.

21. Rexroad CE, Vallejo RL: Estimates of linkage disequilibrium and effective population size in rainbow trout. BMC Genet 2009, 10:83.

22. Sanchez CC, Smith TP, Wiedmann RT, Vallejo RL, Salem M, Yao J, Rexroad CE: Single nucleotide polymorphism discovery in rainbow trout by deep sequencing of a reduced representation library. BMC Genomics 2009, 10:559.

23. Govoroun M, Le GF, Guiguen Y: Generation of a large scale repertoire of Expressed Sequence Tags (ESTs) from normalised rainbow trout CDNA libraries. BMCGenomics 2006, 7:196.

24. Rexroad CE, Lee Y, Keele JW, Karamycheva S, Brown G, Koop B, Gahr SA, Palti Y, Quackenbush J: Sequence analysis of a rainbow trout CDNA library and creation of a gene index. Cytogenet Genome Res 2003, 102:347-354.

25. Rise ML, von Schalburg KR, Brown GD, Mawer MA, Devlin RH, Kuipers N, Busby M, Beetz-Sargent M, Alberto R, Gibbs AR, et al: Development and application of a salmonid EST database and CDNA microarray: data mining and interspecific hybridization characteristics. Genome Res 2004, 14:478-490.

26. Salem M, Kenney PB, Rexroad lii CE, Yao J: Development of a 37 k highdensity oligonucleotide microarray: a new tool dor functional genome research in rainbow trout. J Fish Biol 2008, 72:2187-2206.

27. Katagiri T, Asakawa S, Minagawa S, Shimizu N, Hirono I, Aoki T: Construction and characterization of BAC libraries for three fish species; rainbow trout, carp and tilapia. Anim Genet 2001, 32:200-204.

28. Palti Y, Luo MC, Hu Y, Genet C, You FM, Vallejo RL, Thorgaard GH, Wheeler PA, Rexroad CE: A first generation BAC-based physical map of the rainbow trout genome. BMC Genomics 2009, 10:462.

29. Venter JC, Smith $\mathrm{HO}$, Hood L: A new strategy for genome sequencing. Nature 1996, 381:364-366

30. Larkin DM, Everts-van der Wind A, Rebeiz M, Schweitzer PA, Bachman S, Green C, Wright CL, Campos EJ, Benson LD, Edwards J, et al: A cattle- human comparative map built with cattle BAC-ends and human genome sequence. Genome Res 2003, 13:1966-1972.

31. Shultz JL, Ali S, Ballard L, Lightfoot DA: Development of a physical map of the soybean pathogen Fusarium virguliforme based on synteny with Fusarium graminearum genomic DNA. BMC Genomics 2007, 8:262.

32. Shultz JL, Kazi S, Bashir R, Afzal JA, Lightfoot DA: The development of BACend sequence-based microsatellite markers and placement in the physical and genetic maps of soybean. Theor Appl Genet 2007, 114:1081-1090.

33. Lai CW, Yu Q, Hou S, Skelton RL, Jones MR, Lewis KL, Murray J, Eustice M, Guan P, Agbayani R, et al: Analysis of papaya BAC end sequences reveals first insights into the organization of a fruit tree genome. Mol Genet Genomics 2006, 276:1-12.

34. Xu P, Wang S, Liu L, Peatman E, Somridhivej B, Thimmapuram J, Gong G, Liu Z: Channel catfish BAC-end sequences for marker development and assessment of syntenic conservation with other fish species. Anim Genet 2006, 37:321-326.

35. Boroevich KA, Lubieniecki KP, Chow W, Jong Pd, Schein J, Field M, Moore R, Boer JGd, Koop BF, Davidson WS: Physical mapping and BAC-end sequence analysis of Atlantic Salmon BAC librairies. Plant and Animal Genomes XVI Conference San Diego, CA, USA; 2008.

36. Jurka J, Kapitonov W, Pavlicek A, Klonowski P, Kohany O, Walichiewicz J: Repbase Update, a database of eukaryotic repetitive elements. Cytogenet Genome Res 2005, 110:462-467.

37. de Boer J, Yazawa R, Davidson W, Koop B: Bursts and horizontal evolution of DNA transposons in the speciation of pseudotetraploid salmonids. BMC Genomics 2007, 8:422.

38. Palti Y, Genet C, Luo M-C, Charlet A, Gao G, Hu Y, Castano-Sanchez C, Tabet-Canale K, Krieg F, Yao J, et al: A first generation integrated map of the rainbow trout genome. BMC Genomics 2011, 12:180.

39. Xu P, Li J, Li Y, Cui R, Wang J, Wang J, Zhang Y, Zhao Z, Sun X: Genomic insight into the common carp (Cyprinus carpio) genome by sequencing analysis of BAC-end sequences. BMC Genomics 2011, 12:188.

40. Kuhl H, Beck A, Wozniak G, Canario A, Volckaert F, Reinhardt R: The European sea bass Dicentrarchus labrax genome puzzle: comparative BAC-mapping and low coverage shotgun sequencing. BMC Genomics 2010, 11:68.

41. Kuhl H, Sarropoulou E, Tine M, Kotoulas G, Magoulas A, Reinhardt R: A Comparative BAC Map for the Gilthead Sea Bream (Sparus aurata L.). Journal of Biomedicine and Biotechnology 2011, 2011:1-7.

42. Davidson W, Koop B, Jones S, Iturra P, Vidal R, Maass A, Jonassen I, Lien S, Omholt S: Sequencing the genome of the Atlantic salmon (Salmo salar). Genome Biology 2011, 11:403.

43. Terol J, Naranjo MA, Ollitrault P, Talon M: Development of genomic resources for Citrus clementina: characterization of three deep-coverage BAC libraries and analysis of 46,000 BAC end sequences. BMC Genomics 2008, 9:423.

44. Ewing B, Green P: Base-calling of automated sequencer traces using phred. II. Error probabilities. Genome Res 1998, 8:186-194.

45. Benson G: Tandem repeats finder: a program to analyze DNA sequences. Nucleic Acids Res 1999, 27:573-580.

46. Rozen S, Skaletsky H: Primer3 on the WWW for general users and for biologist programmers. Methods Mol Biol 2000, 132:365-386.

47. Palti Y, Gahr SA, Purcell MK, Hadidi S, Rexroad CE, Wiens GD: Identification, characterization and genetic mapping of TLR7, TLR8a1 and TLR8a2 genes in rainbow trout (Oncorhynchus mykiss). Dev Comp Immunol 2010, 34:219-233.

48. Han Y, Korban SS: An overview of the apple genome through BAC end sequence analysis. Plant Mol Biol 2008, 67:581-588.

49. Dalrymple B, Kirkness E, Nefedov M, McWilliam S, Ratnakumar A, Barris W, Zhao S, Shetty J, Maddox J, O'Grady M, et al: Using comparative genomics to reorder the human genome sequence into a virtual sheep genome. Genome Biology 2007, 8:R152.

doi:10.1186/1471-2164-12-314

Cite this article as: Genet et al:: Analysis of BAC-end sequences in rainbow trout: Content characterization and assessment of synteny between trout and other fish genomes. BMC Genomics 2011 12:314. 